\title{
A New Strategy for Calixindole Formation: Synthesis of a Calix[3]indole with 2,2; 7,2; 7,7-Methylene Linkages and a New Calix[4]indole with 2,2; 7,2; 7,7; 2,7-Methylene Linkages
}

\author{
Kittiya Somphol, Rui Chen, Mohan Bhadbhade, Naresh Kumar, David StC. Black* \\ School of Chemistry, The University of New South Wales, UNSW, Sydney NSW 2052, Australia \\ Fax +61(2)93856141; E-mail: d.black@unsw.edu.au \\ Received: 05.11.2012; Accepted: 19.11.2012
}

\begin{abstract}
A new strategy of acid-catalysed reactions of indole methanols with a 3-aryl-4,6-dimethoxyindole-7-aldehyde leads to an efficient synthesis of a calix[3]indole with 2,$2 ; 7,2 ; 7,7$-links. A new calix[4]indole containing four indole units with 2,$2 ; 7,2 ; 7,7$; 2,7-links has also been prepared by this strategy.
\end{abstract}

Key words: indoles, diindolylmethanes, macrocyclic compounds, acid-catalysed reactions, ipso-substitution reactions, formaldehyde extrusion

Macrocycles containing pyrroles are well established in the field of supramolecular chemistry for the reason that the pyrrole NH groups readily form hydrogen bonds with guest molecules or on deprotonation the nitrogen atoms can bind to metal ions. ${ }^{1-3}$ Two well-known systems are calix[4]pyrroles and porphyrins. ${ }^{4,5}$ However macrocycles containing indoles have not been widely studied in this context, despite the similar acidity of an indole $\mathrm{NH}$ group to that of a pyrrole. The expansion of the five-membered pyrrole ring system to the larger and more highly conjugated indole system creates new synthetic and structural possibilities with great potential for supramolecular chemistry.

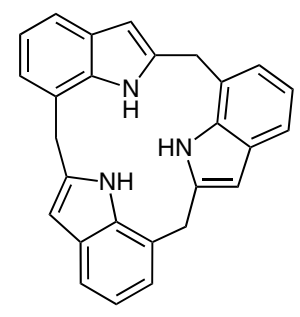

2,$7 ; 2,7 ; 2,7$

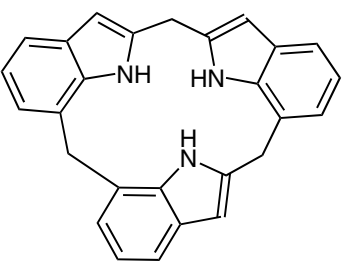

2,$2 ; 7,2 ; 7,7$
Figure 1 Possible structural frameworks of calix[3]indoles

The synthesis of 3-substituted 4,6-dimethoxyindoles has enabled their enhanced activity at $\mathrm{C} 2$ and $\mathrm{C} 7$ to be investigated in a variety of ways. ${ }^{6-11}$ Acid-catalysed reactions of these indoles with aryl aldehydes have led to the formation of calix[3]indoles, presumably via intermediate indole methanols. ${ }^{12}$

SYNLETT 2013, 24, 0024-0028

Advanced online publication: 10.12 .2012

DOI: 10.1055/s-0032-1317858; Art ID: ST-2012-D0947-L

(C) Georg Thieme Verlag Stuttgart · New York
In principle, these calix[3]indoles can contain either three arylmethylene linkages between $\mathrm{C} 2$ and $\mathrm{C} 7$, or one such linkage between $\mathrm{C} 2$ and $\mathrm{C} 2, \mathrm{C} 2$ and $\mathrm{C} 7$, and $\mathrm{C} 7$ and $\mathrm{C} 7$ (see Figure 1). Both types have already been synthesised.

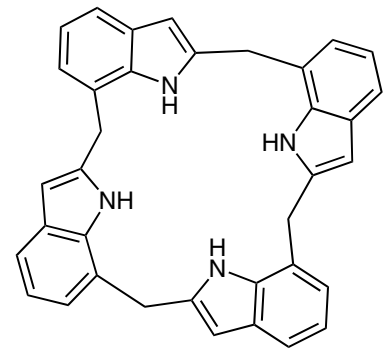

2,$7 ; 2,7 ; 2,7 ; 2,7$

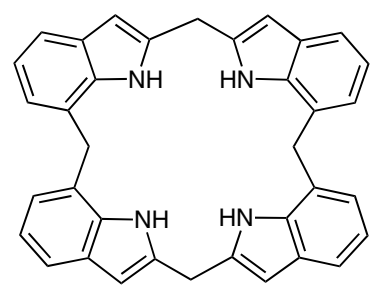

2,$2 ; 7,7 ; 2,2 ; 7,7$

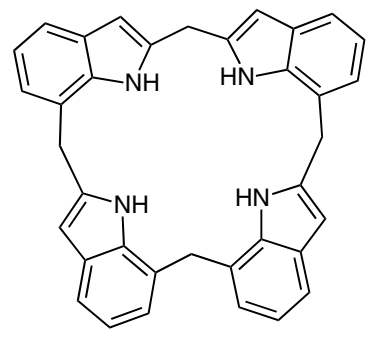

2,$2 ; 7,2 ; 7,7 ; 2,7$

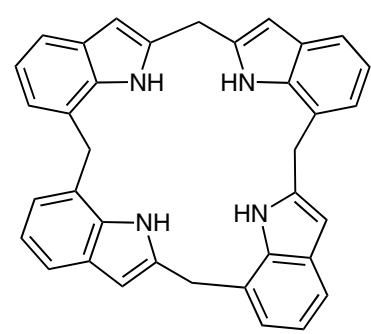

2,$2 ; 7,2 ; 7,2 ; 7,7$
Figure 2 Possible structural frameworks of calix[4]indoles

Both 3-substituted 4,6-dimethoxyindole-7-carbaldehydes and 4,6-dimethoxyindole-2-carbaldehydes can be prepared via the Vilsmeier-Haack formylation reaction. ${ }^{13}$ Subsequent reduction of the formyl groups with sodium borohydride affords the simple indole C7- and C2-methanols, which can also undergo acid-catalysed cyclisation to calix[3] indoles. ${ }^{14-17}$

However, in this process, calix[4]indoles can also be generated, and show four methylene linkages between C2 and C7. There are four possible ways of linking four indoles through the $\mathrm{C} 2$ and $\mathrm{C} 7$ positions with four methylene links. These basic structures are shown in Figure 2.

We now report new, highly effective stepwise sequences for the synthesis of a calix[3]indole and the first example of a calix[4]indole with a 2,2; 7,2; 7,7; 2,7 set of methylene linkages.

These stepwise sequences involve the monoindole 2,7-dimethanol 1 and the diindolyl 7,7'-dimethanol 2, which have been previously reported. ${ }^{14}$ Reaction of dimethanol 
<smiles>[2H]Oc1cc(OC)c(CO)c2[nH]c(CO)c([2H])c12</smiles><smiles>COc1cc(OC)c2c(O)c[nH]c2c1C=O</smiles><smiles>C=C</smiles><smiles></smiles><smiles>[2H]c1c(Cc2[nH]c3c(Cc4[nH]c5c(CO)c(OC)cc(OC)c5c4[2H])c(OC)cc(OC)c3c2[2H])[nH]c2c(CO)c(OC)cc(OC)c12</smiles>

Scheme 1 Reagents and conditions: (a) $p$-TsOH, $i$-PrOH, r.t., 1 h, $90 \%$; (b) $\mathrm{NaBH}_{4}$, THF, EtOH, r.t., 98\%; (c) p-TsOH, DMSO, r.t., 5 min, $89 \%$.

1 with two equivalents of the indole-7-aldehyde 3 in isopropanol containing $p$-toluenesulfonic acid gave the triindolyldimethane dialdehyde $4,{ }^{18}$ which precipitated from the reaction mixture and was obtained in $90 \%$ yield. Reduction of the dialdehyde 4 with sodium borohydride in a mixture of ethanol and tetrahydrofuran gave the dimethanol 5 in $98 \%$ yield. Treatment of this dimethanol 5 with $p$-toluenesulfonic acid in anhydrous dimethylsulfoxide generated the calix[3]indole $6^{19}$ in $89 \%$ yield (Scheme 1).

Compound $\mathbf{6}$ is a new example of this class of previously reported calixindoles. Its ${ }^{1} \mathrm{H}$ NMR spectrum showed proton resonances for six different methoxy groups at $\delta=$ $3.61,3.62,3.63,3.64,3.73$, and $3.76 \mathrm{ppm}$, and three different methylene resonance singlets at $\delta=3.93,4.15$, and $4.20 \mathrm{ppm}$.

Similar treatment of the 7,7'-dimethanol 2 with two equivalents of the indole-7-carbaldehyde $\mathbf{3}$ in isopropanol containing $p$-toluenesulfonic acid gave the tetraindole dialdehyde $7^{20}$ in $68 \%$ yield (Scheme 2). Reduction of dialdehyde 4 with sodium borohydride in a mixture of ethanol and tetrahydrofuran gave the dimethanol $\mathbf{8}$ in $98 \%$ yield. The calix[4]indole $\mathbf{9}^{21}$ was formed in $69 \%$ yield when the dimethanol $\mathbf{8}$ was treated with $p$-toluenesulfonic acid in anhydrous dimethyl sulfoxide briefly at room temperature.

The new calix[4]indole 9, and the first example of this type, was characterised fully and its NMR spectra were particularly revealing with regard to structural symmetry. Seven different methoxy proton resonances were observed at $\delta=3.44,3.53,3.75,3.80,3.95,3.99$ and 4.01 ppm for the eight methoxy groups. There are three different methylene groups, the protons of which form $\mathrm{AB}$ doublets resonating at $\delta=3.98,3.99$ and $4.16 \mathrm{ppm}$, and four different $\mathrm{H} 5$ protons at $\delta=5.91,6.26,6.33$ and $6.48 \mathrm{ppm}$. Furthermore an X-ray crystal structure was obtained (see Figure 3), and showed a rigid cube-like structure, similar to that of a calix[4]indole with four 2,7-methylene links. ${ }^{15}$ It is noteworthy that both types of calix[4]indoles pre-

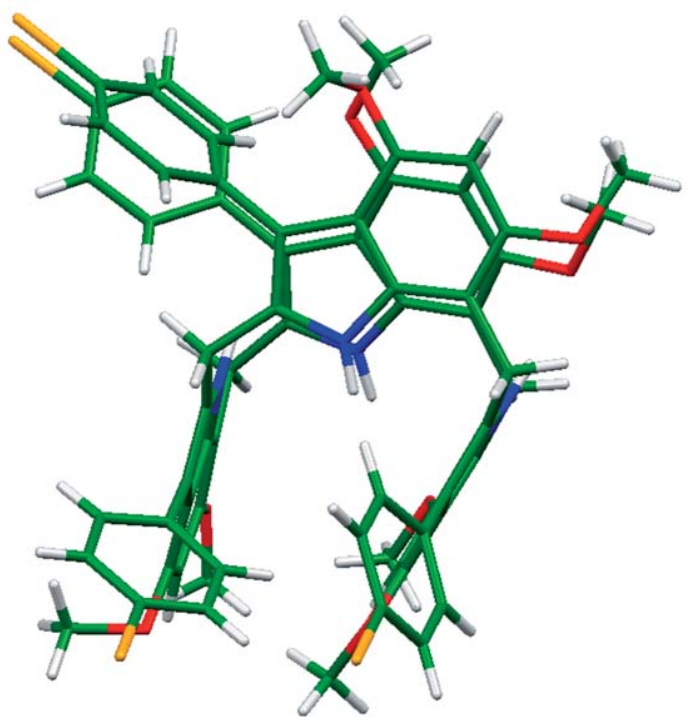

Figure 3 ORTEP diagram of calix[4]indole 9 


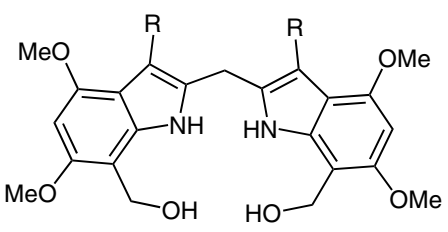<smiles>[R]c1c[nH]c2c(C=O)c(OC)cc(OC)c12</smiles><smiles>[2H]c1c(C/C(C(=C)OC)=C(\C)N)[nH]c2c(C=O)c(OC)cc(OC)c12</smiles>
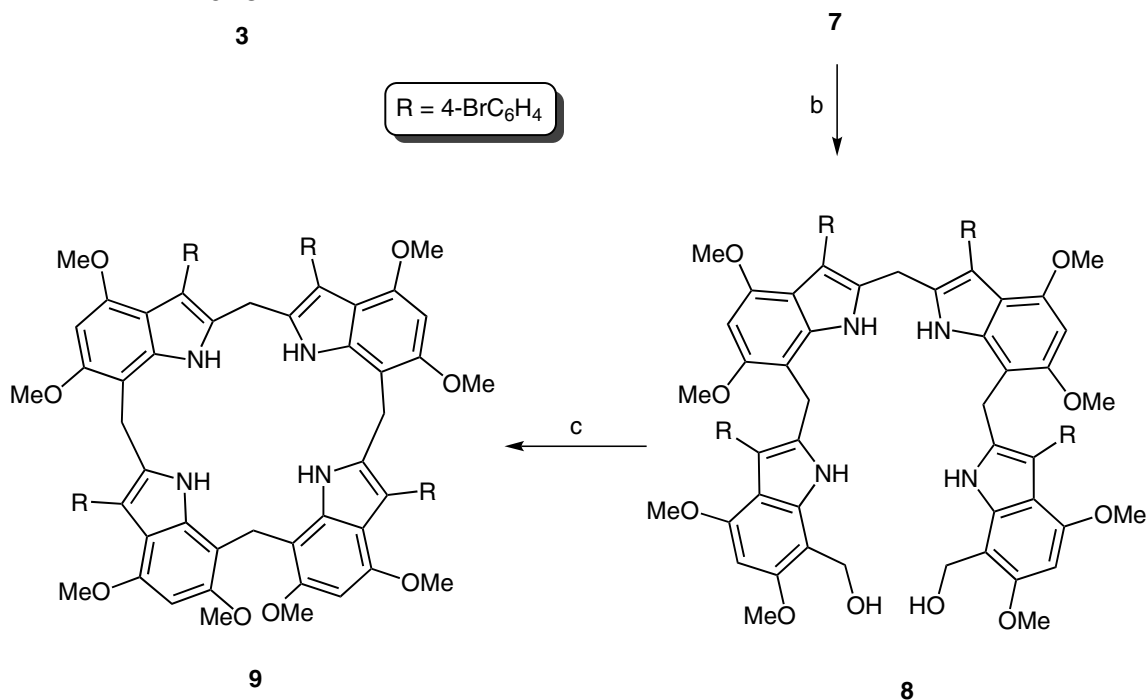

Scheme 2 Reagents and conditions: (a) p-TsOH, $i$-PrOH, r.t., 1 h, 68\%; (b) $\mathrm{NaBH}_{4}$, THF, EtOH, r.t. 98\%; (c) p-TsOH, DMSO, r.t., 5 min, 69\%.

pared so far show rigid structures leading to non-identical methylene protons, whereas the calix[3]indoles, such as compound $\mathbf{6}$ are quite flexible, allowing their methylene link protons to equilibrate on the NMR time scale and show singlet resonances.

The cyclisation reactions of compound 5 to 6 and of compound $\mathbf{8}$ to 9 are typical of 4,6-dimethoxyindoles that are substituted at both $\mathrm{C} 2$ and $\mathrm{C} 3$ and bear a hydroxymethyl group at $\mathrm{C} 7$. This process involves a mildly acid-catalysed ipso-substitution in which the restoration of the indole aromaticity requires the loss of formaldehyde. A postulated mechanism, exemplified for the conversion of compound $\mathbf{8}$ to macrocycle $\mathbf{9}$, is shown in Scheme 3. Although the indole $\mathrm{C} 5$ position is unsubstituted, it is protected against electrophilic substitution by the adjacent methoxy groups, which are in turn buttressed by the $\mathrm{C} 3$ and $\mathrm{C} 7$ substituents. We have observed many examples of this remarkably effective and irreversible formaldehyde extrusion process for the formation of 7,7'-diindolylmethanes. The formation of compounds $\mathbf{6}$ and $\mathbf{9}$ demonstrate the effectiveness of this synthetic strategy for macrocyclisation reactions.

In summary, this synthetic methodology has the capacity to generate calix[3]indoles containing one 2,2-, one 7,2- and one 7,7-link, and calix[4]indoles containing one 2,2-, one 7,7-, and two 7,2-links. Although all four substituents at $\mathrm{C} 3$ are the same in the case of compounds $\mathbf{6}$ and $\mathbf{9}$, there is the possibility to introduce two different substituents by this stepwise procedure. Furthermore, although the indole methanols $\mathbf{1}$ and $\mathbf{2}$ undergo ready reaction with 3 -substituted 4,6-dimethoxyindoles, ${ }^{14}$ linkages occur at both $\mathrm{C} 2$ and $\mathrm{C} 7$ and product mixtures result. The use of the 7-aldehyde 3 provides much cleaner and regiospecific linkage formation.

\section{Acknowledgment}

Research support from the Australian Research Council (Project number: DP1095159) and the University of New South Wales is gratefully acknowledged.

\section{References and Notes}

(1) Gale, P. A.; Sessler, J. L.; Král, V.; Lynch, V. J. Am. Chem. Soc. 1996, $118,5140$.

(2) Allen, W. E.; Gale, P. A.; Brown, C. T.; Lynch, V. M.; Sessler, J. L. J. Am. Chem. Soc. 1996, 118, 12471.

(3) Davis, N. K. S.; Thompson, A. L.; Anderson, H. L. J. Am. Chem. Soc. 2010, 133, 30.

(4) Gale, P. A.; Sessler, J. L.; Kral, V. Chem. Commun. 1998, 1. 

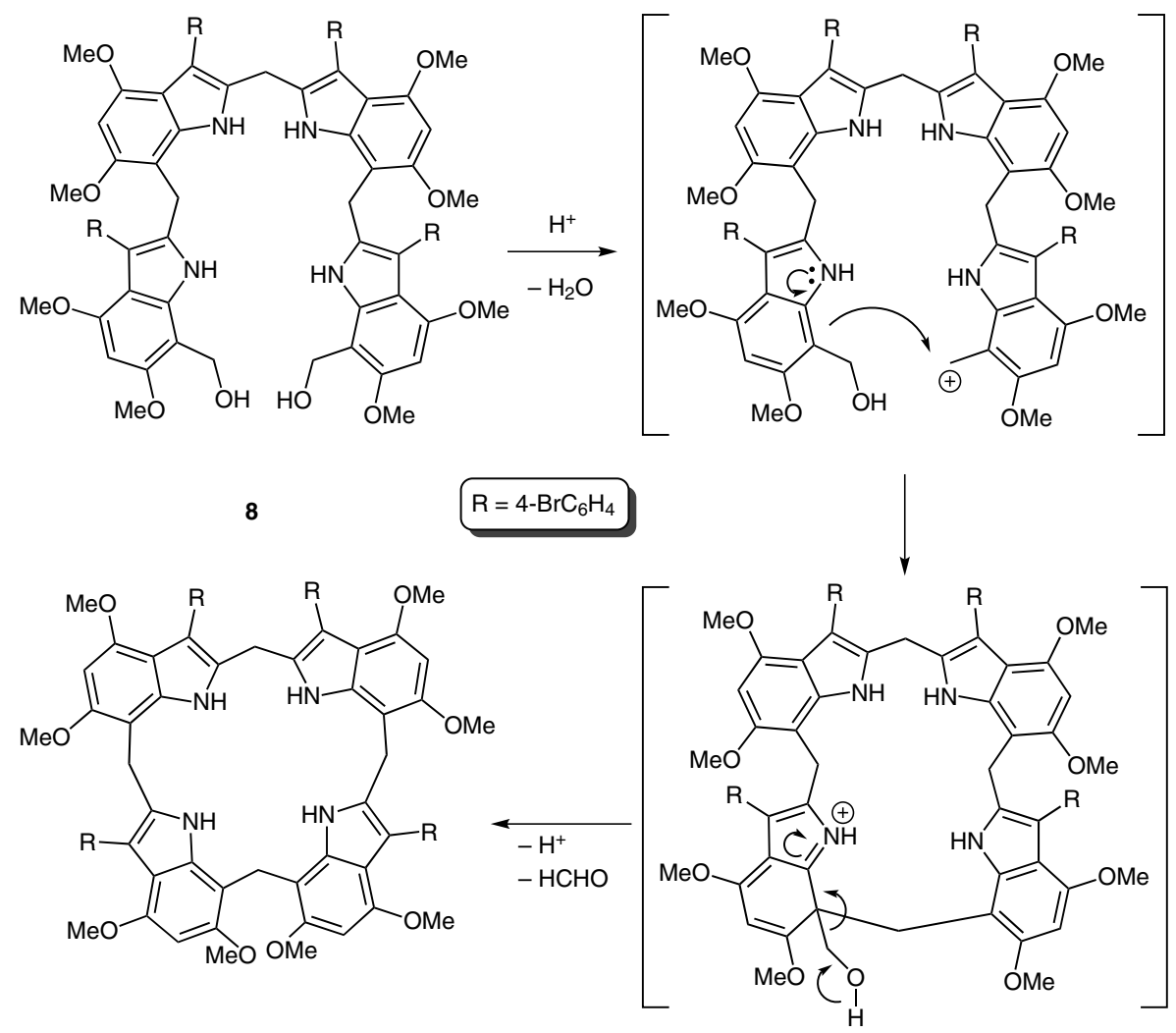

9

Scheme 3 Postulated mechanism for the formation of compound 9

(5) Kurotobi, K.; Kim, K. S.; Noh, S. B.; Kim, D.; Osuka, A. Angew. Chem. Int. Ed. 2006, 45, 3944.

(6) Black, D. StC.; Gatehouse, B. M. K. C.; Theobald, F.; Wong, L. C. H. Aust. J. Chem. 1980, 33, 343.

(7) Black, D. StC.; Rothnie, N. E.; Wong, L. C. H. Tetrahedron Lett. 1980, 21, 1883.

(8) Black, D. StC.; Rothnie, N. E.; Wong, L. C. H. Aust. J. Chem. 1983, 36, 2407.

(9) Black, D. StC.; Kumar, N.; Wong, L. C. H. Aust. J. Chem. 1986, 39, 15.

(10) Black, D. StC.; Bowyer, M. C.; Bowyer, P. K.; Ivory, A. J.; Kim, M.; Kumar, N.; McConnell, D. B.; Popiolek, M. Aust. J. Chem. 1994, 47, 1741.

(11) Pchalek, K.; Jones, A. W.; Wekking, M. T.; Black, D. StC. Tetrahedron 2005, 61, 77.

(12) Black, D. StC.; Craig, D. C.; Kumar, N. Aust. J. Chem. 1996, 49, 311.

(13) Black, D. StC.; Bowyer, M. C.; Catalano, M. M.; Ivory, A. J.; Keller, P. A.; Kumar, N.; Nugent, S. J. Tetrahedron 1994, 50, 10497.

(14) Black, D. StC.; Bowyer, M. C.; Kumar, N.; Mitchell, P. S. R. J. Chem. Soc., Chem. Commun. 1993, 819.

(15) Black, D. StC.; Craig, D. C.; Kumar, N. Tetrahedron Lett. 1995, 36, 8075.

(16) Black, D. StC.; Craig, D. C.; Kumar, N.; McConnell, D. B. Tetrahedron Lett. 1996, 37, 241.

(17) Black, D. StC.; Kumar, N.; McConnell, D. B. Tetrahedron 2000, 56, 8513 .

(18) Compound $\mathbf{4}$ was prepared from the indole-7-aldehyde 3 (0.900 g, $2.50 \mathrm{mmol})$, diindolyl dimethanol 1 (0.490 g, 1.25 $\mathrm{mmol}), p$-toluenesulfonic acid monohydrate and isopropanol $(100 \mathrm{~mL})$. After stirring for $1 \mathrm{~h}$, filtration and drying, the compound $7(1.21 \mathrm{~g}, 90 \%)$ was obtained as a yellow solid; mp 256-257 ${ }^{\circ} \mathrm{C}\left(\mathrm{CH}_{2} \mathrm{Cl}_{2}\right.$-light petroleum). ${ }^{1} \mathrm{H}$ NMR (300
$\left.\mathrm{MHz}, \mathrm{CDCl}_{3}\right): \delta=3.66,3.70,3.82,3.95,3.99,4.07(6 \times \mathrm{s}$, $18 \mathrm{H}, \mathrm{OMe}$ ), 3.82 (s, $2 \mathrm{H}, \mathrm{CH}_{2}, 2,2^{\prime}$-link), 4.18 (s, $2 \mathrm{H}, \mathrm{CH}_{2}$, 2,7'-link), 6.05, 6.15, 6.30 (3 × s, $3 \mathrm{H}$, indolyl H5), 6.91 (s, 1 $\mathrm{H}, \mathrm{NH}), 6.96-7.32(\mathrm{~m}, 12 \mathrm{H}, \mathrm{ArH}), 9.98,10.59(2 \times \mathrm{s}, 2 \mathrm{H}$, $\mathrm{NH}), 10.28,10.32(2 \times \mathrm{s}, 2 \mathrm{H}, \mathrm{CHO}) .{ }^{13} \mathrm{C} \mathrm{NMR}(75 \mathrm{MHz}$, $\left.\mathrm{CDCl}_{3}\right): \delta=20.9,23.7\left(\mathrm{CH}_{2}\right), 55.1,55.2,55.3,56.3,56.4$, 56.7 (OMe), 86.6, 86.8, 89.1 (indolyl C5), 130.0, 130.3, 130.7, 132.1, 132.2, 132.4 (ArCH), 101.3, 104.2, 104.3, $110.9,111.2,111.9,113.5,114.6,119.7,119.9,120.1$, 129.1, 130.5, 130.7, 132.1 (2×), 133.6, 134.0, 134.3, 134.4, 136.3, 153.0, 153.3, 160.2, 160.5, 162.3, 162.4 (ArC), 187.8, 188.0 (CHO). IR (KBr): 3409, 3342, 2934, 2843, 1643, $1593,1562,1511,1487,1464,1434,1393,1367,1353$, $1247,1214,1120,994,817,794 \mathrm{~cm}^{-1}$. UV-Vis $\left(\mathrm{CH}_{2} \mathrm{Cl}_{2}\right)$ : $\lambda_{\max }=234\left(\varepsilon=83,900 \mathrm{~cm}^{-1} \mathrm{M}^{-1}\right), 257(72,400), 317(35,200)$ nm. MS (ESI): $m / z(\%)=1074\left[\mathrm{M},{ }^{79 / 79 / 79} \mathrm{Br}\right](10), 862(15)$, 796 (20), 663 (80), 647 (30), 429 (60), 135 (100), 108 (70). Anal. Calcd for $\mathrm{C}_{52} \mathrm{H}_{42} \mathrm{Br}_{3} \mathrm{~N}_{3} \mathrm{O}_{8}: \mathrm{C}, 58.0 ; \mathrm{H}, 3.9 ; \mathrm{N}, 3.9$. Found: C, 57.9; $\mathrm{H}, 4.0 ; \mathrm{N}, 3.7$.

(19) Compound 6 was prepared by treatment of the triindolyl dimethanol $8(0.300 \mathrm{~g}, 0.280 \mathrm{mmol})$ in anhyd dimethylsulfoxide $(5 \mathrm{~mL})$ with a catalytic amount of $p$ toluenesulfonic acid monohydrate and stirred at r.t. After 5 min, $\mathrm{H}_{2} \mathrm{O}$ was added and the resulting green precipitate was filtered off, washed with $\mathrm{H}_{2} \mathrm{O}$, dried and recrystallised from EtOAc-hexane to yield the calixindole $9(0.26 \mathrm{~g}, 89 \%)$ as a yellow-green solid; $\mathrm{mp}>300{ }^{\circ} \mathrm{C}$. ${ }^{1} \mathrm{H}$ NMR $[300 \mathrm{MHz}$, $\left.\left(\mathrm{CD}_{3}\right)_{2} \mathrm{SO}\right]: \delta=3.61,3.62,3.63,3.64,3.73,3.76(6 \times \mathrm{s}, 18$ $\mathrm{H}, \mathrm{OMe}), 3.93,4.15,4.20\left(3 \times \mathrm{s}, 6 \mathrm{H}, \mathrm{CH}_{2}\right), 6.33,6.34(2 \times$ $\mathrm{s}, 3 \mathrm{H}$, indolyl H5), 7.10-7.15 (m, $4 \mathrm{H}, \mathrm{ArH}), 7.31-7.36(\mathrm{~m}$, $4 \mathrm{H}, \mathrm{ArH}), 7.45$ (d, $J=8.3 \mathrm{~Hz}, 2 \mathrm{H}, \mathrm{ArH}), 7.54$ (d, $J=8.3$ $\mathrm{Hz}, 2 \mathrm{H}, \mathrm{ArH}), 9.49,9.62,9.95(3 \times \mathrm{s}, 3 \mathrm{H}, \mathrm{NH}) .{ }^{13} \mathrm{C} \mathrm{NMR}$ [75 MHz, $\left.\left(\mathrm{CD}_{3}\right)_{2} \mathrm{SO}\right]: \delta=19.2,21.6,23.3\left(\mathrm{CH}_{2}\right), 55.3,55.4$, 55.5, 56.8, 57.4, 57.7 (OMe), 89.7, 90.5, 90.8 (C5), 125.9, 
$128.4,130.1,132.9,133.2,133.5(\mathrm{ArCH}), 102.0,104.3$, 105.0, 111.4, $111.8(2 \times), 114.0,119.1,131.4,132.1,132.3$, 132.9, 134.8, 135.2, 135.4, 135.6, 136.1, 136.7, 137.0, $138.0,152.0(2 \times), 152.3,152.5,153.3,154.1,154.3(\mathrm{ArC})$. IR (KBr): 3419, 2933, 2835, 1619, 1595, 1520, 1488, 1463, 1434, 1393, 1341, 1295, 1207, 1141, 1112, 1073, 997, 819 $\mathrm{cm}^{-1}$. UV-Vis $\left(\mathrm{CH}_{2} \mathrm{Cl}_{2}\right): \lambda_{\max }=233\left(\varepsilon=111,200 \mathrm{~cm}^{-1} \mathrm{M}^{-}\right)$, $289(41,800) \mathrm{nm}$. MS (ESI): $m / z(\%)=1036[\mathrm{M}+1$, $\left.{ }^{81 / 81 / 81} \mathrm{Br}\right](24), 1034\left[\mathrm{M}+1,{ }^{79 / 81 / 81} \mathrm{Br}\right]$ (43), $1032[\mathrm{M}+1$, $\left.{ }^{79 / 79 / 81} \mathrm{Br}\right](20), 1030\left[\mathrm{M}+1,{ }^{79 / 79 / 79} \mathrm{Br}\right]$ (20). Anal. Calcd for $\mathrm{C}_{51} \mathrm{H}_{42} \mathrm{Br}_{3} \mathrm{~N}_{3} \mathrm{O}_{6} \cdot 1.5 \mathrm{H}_{2} \mathrm{O}: \mathrm{C}, 57.8 ; \mathrm{H}, 4.3 ; \mathrm{N}, 4.0$. Found: $\mathrm{C}$, $57.8 ; \mathrm{H}, 4.2 ; \mathrm{N}, 3.9$.

(20) Compound 7 was prepared from the indole-7-aldehyde 3 (49.0 $\mathrm{mg}, 0.136 \mathrm{mmol})$, diindolyl dimethanol $2(50.0 \mathrm{mg}$, $0.068 \mathrm{mmol}), p$-toluenesulfonic acid monohydrate and isopropanol $(30 \mathrm{~mL})$. After stirring for $1 \mathrm{~h}$, the yellowish precipitate was filtered off to yield compound $7(66.0 \mathrm{mg}$, $68 \%$ ); mp $292{ }^{\circ} \mathrm{C}$ (dec.; $\mathrm{CH}_{2} \mathrm{Cl}_{2}$-light petroleum). ${ }^{1} \mathrm{H}$ NMR (300 MHz, $\mathrm{CDCl}_{3}$ ): $\delta=3.57$ (s, $2 \mathrm{H}, \mathrm{CH}_{2}, 2,2^{\prime}$-link), 3.63, 3.70, 3.94, $4.08(4 \times \mathrm{s}, 24 \mathrm{H}, \mathrm{OMe}), 4.07\left(\mathrm{~s}, 4 \mathrm{H}, \mathrm{CH}_{2}, 2,7^{\prime}-\right.$ link), 5.99, $6.36(2 \times \mathrm{s}, 4 \mathrm{H}$, indolyl H5), $6.82(\mathrm{~s}, 2 \mathrm{H}, \mathrm{NH})$, 6.85-6.89 (m, $6 \mathrm{H}, \mathrm{ArH}), 6.98-7.01$ (m, $6 \mathrm{H}, \mathrm{ArH}), 7.17$ (d, $J=8.3 \mathrm{~Hz}, 4 \mathrm{H}, \mathrm{ArH}$ ), 10.26 (s, $2 \mathrm{H}, \mathrm{CHO}$ ), 10.53 (s, $2 \mathrm{H}$, $\mathrm{NH}) .{ }^{13} \mathrm{C}$ NMR $\left(75 \mathrm{MHz}, \mathrm{CDCl}_{3}\right): \delta=21.0,23.9\left(\mathrm{CH}_{2}\right)$, 55.0, 55.2, 56.2, 56.9 (OMe), 86.7, 89.3 (indolyl C5), 129.9, 130.4, 132.1, 132.3 (ArCH), 101.3, 104.1, 110.8, 112.1, $112.5,114.5,119.4,120.0,129.2,132.2,133.8(2 \times), 134.0$, 136.2, 152.8, 153.3, 160.3, 162.3 (ArC), 188.0 (CHO). IR (KBr): 3414, 2934, 2839, 1722, 1644, 1591, 1561, 1517 , 1487, 1464, 1434, 1393, 1367, 1352, 1327, 1291, 1250 , $1213,1119,1071,993,817,794 \mathrm{~cm}^{-1}$. UV-Vis $\left(\mathrm{CH}_{2} \mathrm{Cl}_{2}\right)$ : $\lambda_{\max }=239\left(\varepsilon=147,800 \mathrm{~cm}^{-1} \mathrm{M}^{-1}\right), 312(55,800) \mathrm{nm} . \mathrm{MS}$ (MALDI): $m / z(\%)=1137(100), 1059$ (25). Anal. Calcd for $\mathrm{C}_{69} \mathrm{H}_{56} \mathrm{Br}_{4} \mathrm{~N}_{4} \mathrm{O}_{10} \cdot \mathrm{H}_{2} \mathrm{O}: \mathrm{C}, 57.6 ; \mathrm{H}, 4.1 ; \mathrm{N}, 3.9$. Found: $\mathrm{C}$, $57.5 ; \mathrm{H}, 4.3 ; \mathrm{N}, 3.6$

(21) Compound 9 was prepared by treatment of the tetraindolyl dimethanol 8 (100 $\mathrm{mg}, 0.070 \mathrm{mmol})$ in anhyd dimethylsulfoxide $(5 \mathrm{~mL})$ with a catalytic amount of $p$ toluenesulfonic acid monohydrate and stirred at r.t. After 5 min, $\mathrm{H}_{2} \mathrm{O}$ was added and the resulting yellow-green precipitate was filtered off, washed with $\mathrm{H}_{2} \mathrm{O}$, dried and recrystallised from EtOAc-hexane to yield the calixindole 9 (67.0 $\mathrm{mg}, 69 \%$ ) as a pale yellow solid; $\mathrm{mp}>300{ }^{\circ} \mathrm{C} .{ }^{1} \mathrm{H}$ NMR $\left(300 \mathrm{MHz}, \mathrm{CDCl}_{3}\right): \delta=3.44,3.53,3.75,3.80(2 \times), 3.95$, $3.99,4.01(8 \times \mathrm{s}, 24 \mathrm{H}, \mathrm{OMe}), 3.98(\mathrm{~d}, J=12.7 \mathrm{~Hz}, 2 \mathrm{H}$, $\left.\mathrm{CH}_{2}\right), 3.99\left(\mathrm{~d}, J=16.0 \mathrm{~Hz}, 2 \mathrm{H}, \mathrm{CH}_{2}\right), 4.16(\mathrm{~d}, J=13.7 \mathrm{~Hz}$, $\left.4 \mathrm{H}, \mathrm{CH}_{2}\right), 5.91,6.26,6.33,6.48(4 \times \mathrm{s}, 4 \mathrm{H}$, indolyl H5), $6.95(\mathrm{~d}, J=8.3 \mathrm{~Hz}, 4 \mathrm{H}, \mathrm{ArH}), 7.03(\mathrm{~d}, J=8.3 \mathrm{~Hz}, 4 \mathrm{H}, \mathrm{ArH})$, 7.24 (d, $J=8.3 \mathrm{~Hz}, 4 \mathrm{H}, \mathrm{ArH}), 7.40$ (d, $J=8.3 \mathrm{~Hz}, 4 \mathrm{H}, \mathrm{ArH})$, $8.31,8.81(2 \times \mathrm{s}, 4 \mathrm{H}, \mathrm{NH}) .{ }^{13} \mathrm{C} \mathrm{NMR}\left(75 \mathrm{MHz}, \mathrm{CDCl}_{3}\right): \delta=$ 21.3, 21.5, $29.6\left(\mathrm{CH}_{2}\right), 55.2,55.5,57.4,57.5(\mathrm{OMe}), 88.8$, 89.8 (C5), 130.3, 130.7, 132.1, 132.7 ( $\mathrm{ArCH}), 102.3,111.8$, $112.0,112.3,119.3,119.7,119.9,130.5,132.5,136.2$, 136.6, 136.9, 137.0, $152.2(2 \times), 152.3,152.5,152.6(\mathrm{ArC})$. IR (KBr): 3415, 2933, 2837, 1620, 1596, 1489, 1463, 1434, $1342,1296,1257,1213,1147,1114,996 \mathrm{~cm}^{-1}$. UV-Vis $\left(\mathrm{CH}_{2} \mathrm{Cl}_{2}\right): \lambda_{\max }=279 \mathrm{~nm}\left(\varepsilon=111,100 \mathrm{~cm}^{-1} \mathrm{M}^{-}\right) . \mathrm{MS}$ (MALDI): $m / z(\%)=1379\left[\mathrm{M}-1,{ }^{81 / 81 / 81 / 81} \mathrm{Br}\right](27), 1377[\mathrm{M}$ $\left.-1,{ }^{79 / 81 / 81 / 81} \mathrm{Br}\right](70), 1375\left[\mathrm{M}-1,{ }^{79 / 79 / 81 / 81} \mathrm{Br}\right](100), 1373$ $\left[\mathrm{M}-1,{ }^{79 / 79 / 79 / 81} \mathrm{Br}\right](72), 1371\left[\mathrm{M}-1,{ }^{79 / 79 / 79 / 79} \mathrm{Br}\right](35)$. Anal. Calcd for $\mathrm{C}_{68} \mathrm{H}_{56} \mathrm{Br}_{4} \mathrm{~N}_{4} \mathrm{O}_{8}: \mathrm{C}, 59.3 ; \mathrm{H}, 4.1 ; \mathrm{N}, 4.1$. Found: C, 59.6; H, 4.3; N, 4.0.

Single crystals were obtained from a solvent mixture of $\mathrm{CH}_{2} \mathrm{Cl}_{2}$ and EtOAc: $\mathrm{C}_{68} \mathrm{H}_{56} \mathrm{Br}_{4} \mathrm{~N}_{4} \mathrm{O}_{8} \cdot \mathrm{CCl}_{3}, M=1495.17$, monoclinic, $a=25.00(3) \AA, b=19.47(2) \AA, c=12.594(10)$ $\AA, \alpha=90.00^{\circ}, \beta=102.39(3)^{\circ}, \gamma=90.00^{\circ}, V=5986(10) \AA^{3}$, $T=150(2) \mathrm{K}$, space group $C 2 / c, \mathrm{Z}=4, \mu(\mathrm{MoK} \alpha)=2.887$ $\mathrm{mm}^{-1}, 19259$ reflections measured, 5245 independent reflections $\left(R_{\text {int }}=0.2689\right)$. The final $R_{1}$ values were 0.0824 $[I>2 \sigma(I)]$. The final $\mathrm{wR}\left(\mathrm{F}^{2}\right)$ values were $0.0864[I>2 \sigma(I)]$. The final $R_{1}$ values were 0.3272 (all data). The final $\mathrm{wR}\left(\mathrm{F}^{2}\right)$ values were 0.1306 (all data). The goodness of fit on $\mathrm{F}^{2}$ was 0.988 (all data). Crystallographic data for compound 9 has been deposited with the Cambridge Crystallographic Data Centre as supplementary publication No. CCDC 894925. Copies of the data can be obtained free of charge on application to CCDC, 12 Union Road, Cambridge CB21EZ, UK [fax: +44(1223)336033; email: deposit@ccdc.cam.ac.uk]. 